\title{
Social Networks and Crowdsourcing for Stakeholder Analysis in System of Systems Projects
}

\author{
Soo Ling Lim \\ Software Systems Research Centre \\ School of Design, Engineering and Computing \\ Bournemouth University \\ slim@bournemouth.ac.uk
}

\author{
Cornelius Ncube \\ Software Systems Research Centre \\ School of Design, Engineering and Computing \\ Bournemouth University \\ cncube@bournemouth.ac.uk
}

\begin{abstract}
In system of systems engineering, stakeholders are individuals, groups or organizations that stand to gain or lose from the success or failure of a system of systems. Systems of systems pose unique problems for stakeholder analysis due to their evolutionary nature, emergent behavior, operational independence, managerial independence, and geographical distribution of their constituent systems. This paper proposes the use of social networks and crowdsourcing to identify and prioritize the stakeholders for system of systems projects. The proposed method crowdsources the stakeholders to recommend other stakeholders, builds a social network of stakeholders, and prioritizes stakeholders using social network measures. The paper describes the method, and discusses the strengths and limitations of applying the method in system of systems projects.
\end{abstract}

Keywords: System of systems, requirements engineering, stakeholder identification, stakeholder analysis, social network analysis, crowdsourcing

\section{Introduction}

System of systems is a class of complex systems whose constituents are themselves complex [1]. The constituent systems can be loosely coupled and can operate independently to produce products or services that satisfy their customer objectives [2]. In system of systems engineering, stakeholders are individuals, groups or organizations that stand to gain or lose from the success or failure of a system of systems [3]. These stakeholders include stakeholders of the system of systems, as well as stakeholders of the constituent systems, which can have conflicting goals and objectives with one another.

Most, if not all, systems of systems engineering projects involve thousands or even hundreds of thousands of stakeholders, with different stakeholders having different levels of influence in the project [4]. As a result, in addition to identifying stakeholders, a crucial step in stakeholder analysis involves prioritizing stakeholders based on their influence in the project so as to focus efforts on highinfluence stakeholders while still being aware of lowinfluence ones. For groups and organizations, there is also the need to identify suitable representatives to act on their behalf.
A large number of projects fail because they omit stakeholders or involve individuals who lack knowledge, time or interest for adequate project involvement. As system requirements are elicited from stakeholders, omitting stakeholders gives rise to missing requirements, which leads to building the wrong product. For example, a company found that all the change requests during the first year of their system deployment came from stakeholders that were overlooked [5]. Some mistakes can be costly. Another company cancelled their project after realizing that they overlooked a stakeholder, which would have cost them $\$ 20$ million [6]. It is not surprising that finding stakeholders and the right representatives has been a major concern for system designers [7].

Systems of systems pose unique problems for stakeholder analysis due to their evolutionary nature, emergent behavior, operational independence, managerial independence, and geographical distribution of their constituent systems [8-11]. Our experiences as stakeholder analysts and the review of the literature have revealed the following shortcomings with existing stakeholder analysis methods. First, some methods are likely to miss out stakeholders, and others find irrelevant parties or "nonstakeholders." Second, most methods treat all stakeholders as equally influential and all group representatives as equally suitable. These methods may suffice in small or monolithic projects but fall short in system of systems projects due to their inability to cater to the complexity and large number of stakeholders involved.

In this paper, we propose the use of social network analysis and crowdsourcing to identify and prioritize stakeholders of systems of systems. The proposed method crowdsources the stakeholders themselves to help in the stakeholder analysis process, and as a result, is able to scale to the complexity and number of stakeholders commonly found in systems of systems projects.

The rest of the paper is organized as follows. Section 2 reviews existing work. Section 3 describes the proposed method. Section 4 discusses our experiences and lessons learned applying the method in practice and Section 5 concludes. 


\section{Existing Methods}

Existing stakeholder analysis approaches can be classified into semi-structured and checklist-based approaches, interview, and search. Semi-structured approaches identify stakeholders by asking context-free questions such as who are the customers, who interacts with the system, and who has interests in the success or failure of the system $[5,6]$. These approaches largely form the basis of existing practices. However, as the constituent systems of a system of systems can have their own customers and stakeholders, such approaches return a large number of results that are difficult if not impossible to prioritize. In addition, context-free questions elicit broad answers and are likely to miss out stakeholders that are specific to the project $[12,13]$. Checklist-based approaches provide a checklist of generic stakeholder roles to assist in the identification of project specific stakeholders $[13,14]$. Checklists do not provide an exhaustive list of stakeholders for a given project, hence it is possible to omit stakeholders that are not on the checklist [13].

The interview method identifies obvious stakeholders and then interview them to learn about other stakeholders [12]. Each newly identified stakeholder is then interviewed to identify more stakeholders. Although the interview method can be thorough, it requires a large amount of time and resources [13]. As a result, they are difficult to scale to systems of systems projects with large numbers of stakeholders. In addition, the method can be difficult to apply to constituent systems of a system of systems with highly distributed geographical locations, and operational and managerial independence. Finally, the method can identify many stakeholders, but lacks a way to prioritize the stakeholders, which will hinder decision-making [12].

The search method identifies an initial set of stakeholders from system users, developers and legislators, to decision-makers. Additional stakeholders are identified by considering parties who provide information or supporting tasks to these stakeholders, parties who process or inspect their products, and parties who interact or support them and vice versa. This is repeated for each newly identified stakeholder [15]. The search method has the potential to generate too much data and return irrelevant parties or "non-stakeholders" [15]. Similar to the interview method, the search method does not provide a means to prioritize the large number stakeholders it identifies.

Existing stakeholder management tools hold and process the data provided by the experts in charge of stakeholder analysis, but they provide little support in the actual identification and prioritization of stakeholders. For example, using the power and interest grid, the experts make a list of stakeholders and plot them against two variables on a matrix: power and interest. In the Stakeholder Circle tool, experts enter stakeholder information such as name, role, significance, comments about the stakeholder, and whether the stakeholder is active [16]. Based on the information, the tool generates graphs and reports. Both tools suffer from the inability to scale to the large number of stakeholders that a system of systems might have. In addition, prioritization is likely to be biased towards the personal perspective of the analyst [17].

\section{Social Networks and Crowdsourcing for Stakeholder Analysis}

The unique properties of system of systems mean that a dynamic, emergent and adaptive method for stakeholder analysis is required. Social networks have been shown to exhibit many of these properties $[18,19]$. The idea is to be open and inclusive, so that each stakeholder participates in the stakeholder analysis process. As stakeholders are socially related to one another, they can be identified and prioritized using their relations [17]. Because of the complexity of systems of systems and large number of stakeholders involved, it is only with the participation of stakeholders and an intelligent aggregation of all their views that stakeholder analysis for systems of systems can be conducted effectively [17].

The proposed method identifies an initial set of stakeholders, asks the stakeholders to recommend other stakeholders, builds a social network with stakeholders as nodes and their recommendations as links, and prioritizes stakeholders using social network measures such as betweenness centrality and in-degree centrality $[17,20]$. The method is shown to be effective in identifying and prioritizing stakeholders in large software projects [17], and is supported by web-based application that we have developed called StakeSource ${ }^{1}[20,21]$. StakeSource was initially developed for stakeholder analysis in the software domain, but have since been used in non-IT projects [22].

To use StakeSource, the analyst sets up a project via its web interface by providing the project details such as the project scope and constituent systems. The analyst then identifies initial stakeholders and provides StakeSource with their details, such as their name, the role in the project, and email address. StakeSource contacts the initial stakeholders via email, providing them with an overview of the project and inviting them to recommend other stakeholders via its web interface. Each recommendation consists of the stakeholder's name, their role in the project, their level of influence in the project, and their email address. Stakeholders can also comment on the stakeholders they recommend. Public comments can be viewed by all stakeholders; private comments are only available to the analyst.

Each time a new stakeholder is identified, StakeSource contacts the stakeholder to invite them to

1 A demonstration of StakeSource can be found at http://vimeo.com/18250588. 
recommend other stakeholders. This technique is also known as the snowballing technique [19], where the set of stakeholders build up like a snowball rolled down a hill. StakeSource provides an option for "non-stakeholders" or stakeholders who lack time or interest to unsubscribe from the project, as well as suggest relevant stakeholders.

Using the data collected from stakeholders, StakeSource supports stakeholder analysis as follows:

- Stakeholder identification. StakeSource aggregates the stakeholders and roles into a complete stakeholder list. At any time during the project, the analyst can access StakeSource to get an up-to-date list of stakeholders, their details, and comments about them. StakeSource provides two views: a list of stakeholders for the entire system of systems and a list of stakeholders for each constituent system.

- Stakeholder prioritization. StakeSource builds a social network of stakeholders with the stakeholders as nodes, and their recommendations as directed links: $S 1$ links to $S 2$ if $S 1$ recommends $S 2$ to be a stakeholder. Then, it prioritizes the stakeholders using social network measures. For example, indegree centrality prioritizes stakeholders who receive the most recommendations and betweenness centrality prioritizes stakeholders who are widely recommended by disparate groups of stakeholders [21]. Each time a measure is selected, StakeSource applies the measure and displays the prioritized list of stakeholders and their roles in the stakeholder analysis user interface (Figure 1 bottom half). StakeSource prioritizes stakeholders for the entire system of systems and for each constituent system.

- Stakeholder network visualization and stakeholder information. The stakeholders' recommendations are visualized as a social network (Figure 1 top half). StakeSource enables analysts and stakeholders to study a stakeholder's position in the social network, their details, priority in the project, the stakeholders who recommended them, the stakeholders they recommend, and comments from other stakeholders.

- Stakeholder management. StakeSource enables the analysts to communicate with individual stakeholders and groups of stakeholders.

\section{Experiences and Lessons Learned}

In this section, we describe our experiences and lessons learned from using StakeSource in projects that exhibit the characteristics of system of systems as defined by Maier [8, 11]. The projects and their characteristics are summarized in Table 1. Our description focuses on the strengths and limitations of StakeSource in supporting these characteristics, which are specific to systems of systems.

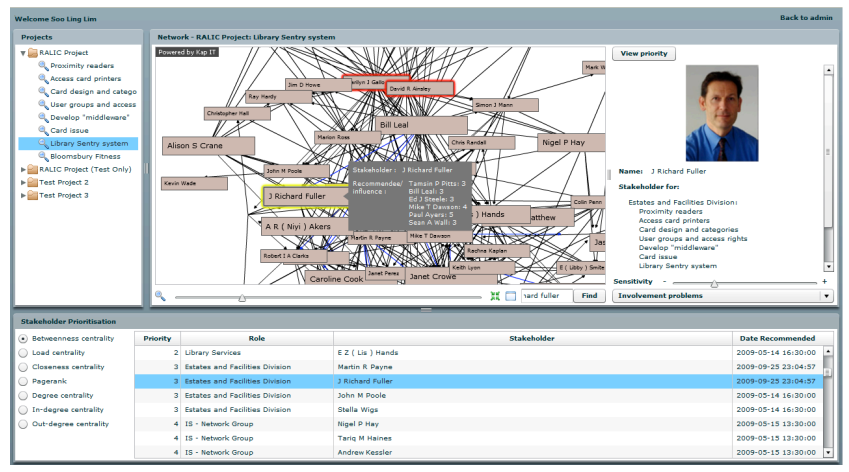

Figure 1. StakeSource stakeholder analysis interface (refer to [21] for enlarged figure).

Table 1. Projects and System of Systems Characteristics

\begin{tabular}{|c|c|c|c|c|c|}
\hline $\begin{array}{l}\text { System of Systems } \\
\text { Project } \\
\text { Description }\end{array}$ & 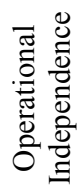 & 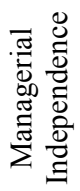 & 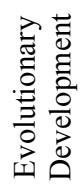 & 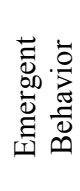 & 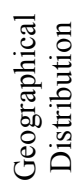 \\
\hline $\begin{array}{l}\text { Project to implement a } \\
\text { nation-wide cloud } \\
\text { computing } \\
\text { infrastructure. }\end{array}$ & $\checkmark$ & $\checkmark$ & & $\checkmark$ & $\checkmark$ \\
\hline $\begin{array}{l}\text { Project to investigate } \\
\text { and propose policy } \\
\text { changes on urban life } \\
\text { and citizen health in } \\
\text { multiple cities. }\end{array}$ & $\checkmark$ & $\checkmark$ & $\checkmark$ & $\checkmark$ & $\checkmark$ \\
\hline $\begin{array}{l}\text { Project to integrate and } \\
\text { aggregate data from } \\
\text { multiple independent } \\
\text { systems to provide an } \\
\text { information service. }\end{array}$ & $\checkmark$ & $\checkmark$ & $\checkmark$ & $\checkmark$ & \\
\hline $\begin{array}{l}\text { Project to investigate } \\
\text { and propose changes to } \\
\text { cross-organizational } \\
\text { practices by a national } \\
\text { run service. }\end{array}$ & $\checkmark$ & $\checkmark$ & $\checkmark$ & $\checkmark$ & $\checkmark$ \\
\hline $\begin{array}{l}\text { Project to integrate } \\
\text { multiple geographically } \\
\text { distant legacy systems } \\
\text { and new systems in the } \\
\text { ICT sector. }\end{array}$ & $\checkmark$ & $\checkmark$ & & $\checkmark$ & $\checkmark$ \\
\hline
\end{tabular}

\subsection{Operational Independence}

A system of systems is composed of systems that are independent and useful in their own right [8]. StakeSource was able to uncover stakeholders in constituent systems that turned out to play important roles in the system of systems integration. StakeSource was reported to be simple to use requiring little or no training from the analysts and stakeholders, which is an important aspect to encourage analyst adoption and stakeholder response. The stakeholder prioritization feature was found to be useful when a large 
number of stakeholders were identified. The stakeholder network diagram provided an overview of the stakeholder relationships within the system of systems and between constituent systems (Figure 2). StakeSource even identified additional constituent systems that should be involved in the system of systems, and in many cases, demonstrated to the system of systems designer that the scope of the project is broader than initially expected.

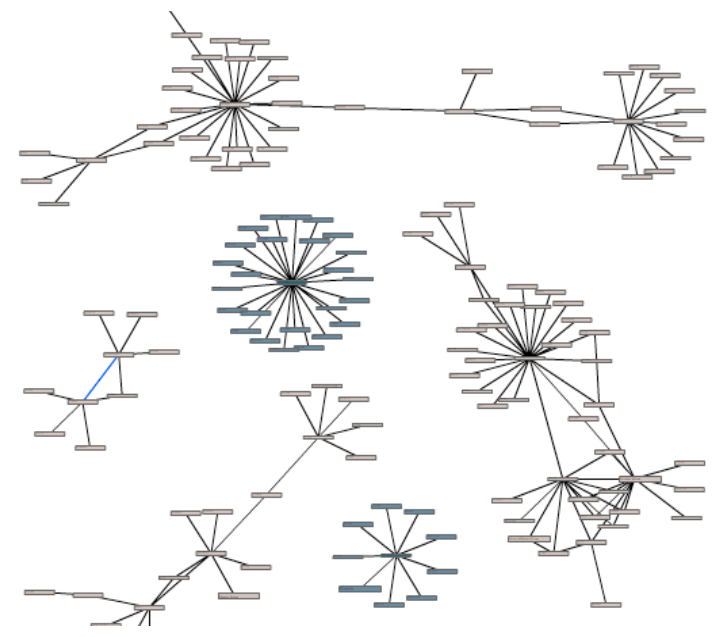

Figure 2. Partial stakeholder network of a project as captured in StakeSource.

StakeSource functions in an open and inclusive manner whereby stakeholders share their knowledge and information about other stakeholders. However, operational independence means that conflicts of interest can exist among constituent systems and the system of systems, resulting in the need for the stakeholders from a constituent system to withhold information from StakeSource, which can be crucial to the successful implementation of the system of systems. As such, StakeSource is well suited for collaborative projects but less suited for high security domains or projects involving highly competing constituent systems. In some projects, StakeSource was able to identify stakeholders who are withholding information and negative stakeholders who are unsupportive of the project. A stakeholder's lack of participation in StakeSource can potentially indicate their lack of participation during system of systems integration. Identifying these parties early in the project can help the designers plan the project better and manage risks.

As systems of systems tend to have unclear boundaries, it impossible to identify all the stakeholders of a system of systems. Stakeholders who were identified can be connected to networks that were not currently mapped; yet stakeholders in those networks can indirectly affect the project. For example, a constituent system can rely on other systems that are, in turn, reliant on other systems and so on. The failure of one of the systems will create a chain reaction that will affect the system of systems. However, resource and time limitations meant that it is impossible and unrealistic to include all these systems in the analysis.
As such, the decision of when to stop looking for stakeholders is a trade-off between time spent identifying the stakeholders and the risk of overlooking a stakeholder. In projects where the consequence of overlooking stakeholders is dire, it is better to identify superfluous ones than to fail to find them all [23]. These projects can extend their snowballing process to get a larger set of stakeholders, but should also expect the stakeholder list to contain more "non-stakeholders."

\subsection{Managerial Independence}

In a system of systems, constituent systems are usually individually acquired and integrated, and they continue to operate independently of the system of systems [8]. The ease of use of StakeSource reduces participation cost thus encouraging responses from stakeholders. However, as the system of systems has very limited amount of centralized control and authority, it can be challenging to motivate stakeholders from constituent systems to respond. In projects where stakeholders provided recommendations, StakeSource was able to produce a comprehensive stakeholder list. However, in some projects, there were little incentives for stakeholders to participate, and as a result the responses collected by StakeSource were insufficient to add value. This shows that the effectiveness of StakeSource depended on the stakeholders' willingness to share information. The successful use of StakeSource for system of systems stakeholder analysis depends on the designer to make the benefit of participating far exceed the cost [8]. Stakeholders were more motivated to respond when an authoritative figure endorsed its use. This is similar to Krygiel's finding on system of systems integration [24]: the process is dependent upon adequate support. Required will be an on-site acquisition leader and empowered to integrate the system of systems, and a system of systems team with sufficient resources and authority to manage the constituent systems [24].

It is conceivable for a constituent system to be part of more than one system of systems. Future work should investigate reusing stakeholder information collected by StakeSource, so that the stakeholders will not be overloaded with repeated requests to nominate stakeholders. While doing so, a challenge is to know which information to reuse and which to update, for example, the level of influence of a stakeholder may be different in different systems of systems.

\subsection{Evolutionary Development}

Development of system of systems is evolutionary over time and with structure, function and purpose added, removed, and modified as experience with the system increases and changes over time [8]. The "unsubscribe" feature in StakeSource revealed stakeholders who have moved on, changed their roles, or no longer have the time or interest to be involved in the project. It is important for 
system of systems designers to be aware of these individuals to assist in their planning. However, although StakeSource provides the facility to add, delete, and modify project scope, constituent systems, and individual stakeholders throughout the lifecycle of the project, it relies on the stakeholders to update information about themselves and other stakeholders, as well as the constituent systems involved and project scope. Future work should investigate methods to motivate or incentivize stakeholders to keep their information up to date, so that the stakeholder network in StakeSource mirrors the evolutionary development of the system of systems.

\subsection{Emergent Behavior}

The system of systems performs functions and carries out purposes that do not reside in any constituent system [8]. These behaviors are emergent properties of the entire system of systems and not the behavior of any constituent system. StakeSource is suited for systems with emergent behavior as the stakeholder network emerges over time mirroring the emergent needs of the system of systems. Other related works have studied the use of stakeholder networks to predict stakeholder involvement in a project [25], the use of genetic algorithms to identify key stakeholders [26], and the use of social networks to identify and prioritize requirements [27, 28]. Emergent properties of systems of systems may result in unanticipated outcomes and affect new stakeholders. We anticipate that the use of crowdsourcing as exploited by StakeSource would be one of the most effective, if not the most effective way of gathering information about new stakeholders. For example, crowdsourcing has been successfully used for disaster relief in the 2010 Haiti earthquake and 2011 Japan tsunami $[29,30]$.

\subsection{Geographical Distribution}

In a system of systems, constituent systems are often geographically dispersed, making it costly to engage with stakeholders [8]. The web-based nature of StakeSource is well suited for supporting stakeholders from multiple locations as stakeholders can provide recommendations in their own time without needing to be physically present. The automated elicitation of stakeholder information was reported to be a significant timesaving and a comparative list of stakeholders was reported by analysts to take weeks to compile manually. However, the differences in culture and social conventions among different countries and organizations influenced the quality of recommendations. For example, stakeholders from reserved cultures were more likely to refrain from commenting on other stakeholders or recommending high status stakeholders. Also, different organizations (or even the same organization in different countries) have different busy periods. Such factors need to be taken into consideration when sourcing stakeholders for help as they influence recommendations and the quality of the stakeholder list returned by StakeSource.

\section{Conclusions}

Systems of systems pose unique problems for stakeholder analysis due to their evolutionary nature, emergent behavior, operational independence, managerial independence, and geographical distribution of their constituent systems. This paper proposes the use of social network analysis and crowdsourcing to identify and prioritize stakeholders of systems of systems. The proposed method identifies an initial set of stakeholders, asks the stakeholders to recommend other stakeholders, builds a social network with stakeholders as nodes and their recommendations as links, and prioritizes stakeholders with social network measures. This method is supported by a web-based tool called StakeSource.

The evaluation of StakeSource on multiple projects exhibiting common characteristics of systems of systems demonstrated that StakeSource is a simple yet effective tool for stakeholder analysis. The stakeholders themselves are involved in stakeholder analysis, and as a result, the method is able to scale to the complexity and number of stakeholders typical in systems of systems projects. We learned that the effectiveness of StakeSource depended on the stakeholders' willingness to share information. As such, systems of systems designers need to understand what motivates stakeholders to contribute.

\section{Acknowledgment}

This work was supported in part by the European Commission Trans-Atlantic Research and Education Agenda in System of Systems Support Action INFSO-ICT287593.

\section{References}

[1] M. Jamshidi, System of Systems Engineering: Innovations for the Twenty-First Century. Wiley, 2011.

[2] M. L. Butterfield, J. S. Pearlman, and S. C. Vickroy, "A system-of-systems engineering GEOSS: Architectural approach," IEEE Systems Journal, vol. 2, pp. 321-332, 2008.

[3] B. Nuseibeh and S. Easterbrook, "Requirements engineering: a roadmap," in Proc. of the Conf. on the Future of Software Enginering, 2000, pp. 35 - 46.

[4] P. Chen and J. Clothier, "Advancing systems engineering for systems-of-systems challenges," Systems Engineering, vol. 6, pp. 170-183, 2003.

[5] A. Cockburn, Writing Effective Use Cases. AddisonWesley Professional, 2000.

[6] D. C. Gause and G. M. Weinberg, Exploring Requirements: Quality Before Design. Dorset House Publishing, 1989. 
[7] I. Alexander and S. Robertson, "Understanding project sociology by modeling stakeholders," IEEE Software, vol. 21, pp. 23-27, 2004.

[8] M. W. Maier, "Architecting principles for systems-ofsystems," Systems Engineering, vol. 1, pp. 267-284, 1998.

[9] C. B. Keating, J. J. Padilla, and K. Adams, "System of systems engineering requirements: challenges and guidelines," Engineering Management Journal, vol. 20, pp. 24-31, 2008.

[10] C. Ncube, "On the Engineering of Systems of Systems: key challenges for the requirements engineering community," in Proc. of the IEEE Workshop on Requirements Engineering for Systems, Services and Systems-of-Systems, 2011, pp. 70-73.

[11]A. P. Sage and C. D. Cuppan, "On the systems engineering and management of systems of systems and federations of systems," Information Knowledge Systems Management, vol. 2, pp. 325-345, 2001.

[12]A. Pouloudi and E. A. Whitley, "Stakeholder identification in inter-organizational systems: gaining insights for drug use management systems," European Journal of Information Systems, vol. 6, pp. 1-14, 1997.

[13]I. Alexander, "A taxonomy of stakeholders: human roles in system development," International Journal of Technology and Human Interaction, vol. 1, pp. 23-59, 2005.

[14]R. E. Freeman, Strategic Management: A Stakeholder Approach. Pitman Publishing, 1984.

[15]H. Sharp, G. H. Galal, and A. Finkelstein, "Stakeholder identification in the requirements engineering process," in Database \& Expert System Applications Workshop, 1999, pp. 387-391.

[16]L. Bourne and D. H. Walker, "Project relationship management and the Stakeholder Circle," International Journal of Managing Projects in Business, vol. 1, pp. 125-130, 2008.

[17] S. L. Lim, D. Quercia, and A. Finkelstein, "StakeNet: using social networks to analyse the stakeholders of large-scale software projects," in Proc. of the ACM/IEEE 32nd International Conf. on Software Engineering (ICSE) - Vol. 1, 2010, pp. 295-304.

[18] S. Wasserman and K. Faust, Social Network Analysis: Methods and Applications. Cambridge University Press, 1994.

[19]J. Scott, Social Network Analysis: A Handbook. Sage, 2000.
[20]S. L. Lim, "Social Networks and Collaborative Filtering for Large-Scale Requirements Elicitation," PhD Thesis, University of New South Wales, Australia, 2010.

[21]S. L. Lim, D. Quercia, and A. Finkelstein, "StakeSource: harnessing the power of crowdsourcing and social networks in stakeholder analysis," in Proc. of the ACM/IEEE 32nd International Conf. on Software Engineering (ICSE) - Vol. 2, 2010, pp. 239242 .

[22] S. L. Lim, D. Damian, F. Ishikawa, and A. Finkelstein, "Using Web 2.0 for stakeholder analysis: StakeSource and its application in ten industrial projects," in Managing Requirements Knowledge: Springer Computer Science Editorial, 2013.

[23]S. Robertson and J. Robertson, Mastering the Requirements Process. Addison-Wesley Professional, 2006.

[24]A. J. Krygiel, "Behind the Wizard's Curtain. An Integration Environment for a System of Systems," DTIC Document, 1999.

[25] S. L. Lim and P. J. Bentley, "Evolving relationships between social networks and stakeholder involvement in software projects," in Proc. of the ACM 13th Genetic and Evolutionary Computation Conf. (GECCO), 2011, pp. 1899-1906.

[26] S. L. Lim, M. Harman, and A. Susi, "Using Genetic Algorithms to Search for Key Stakeholders in LargeScale Software Projects," in Aligning Enterprise, System, and Software Architectures, 2013, pp. 118134.

[27] S. L. Lim and A. Finkelstein, "StakeRare: using social networks and collaborative filtering for large-scale requirements elicitation," IEEE Transactions on Software Engineering, vol. 38, pp. 707-735, 2012.

[28]S. L. Lim, D. Damian, and A. Finkelstein, "StakeSource2.0: using social networks of stakeholders to identify and prioritise requirements," in Proc. of the IEEE 33rd International Conf. on Software Engineering (ICSE), 2011, pp. 1022-1024.

[29] M. Zook, M. Graham, T. Shelton, and S. Gorman, "Volunteered geographic information and crowdsourcing disaster relief: a case study of the Haitian earthquake," World Medical \& Health Policy, vol. 2, pp. 7-33, 2012.

[30]H. Gao, G. Barbier, and R. Goolsby, "Harnessing the crowdsourcing power of social media for disaster relief," IEEE Intelligent Systems, vol. 26, pp. 10-14, 2011. 